\title{
Checkpoint inhibitor pneumonitis in Chinese lung cancer patients: clinical characteristics and risk factors
}

\author{
Chen Zhang ${ }^{1 \#}$, Fangyan Gao ${ }^{1 \#}$, Shidai Jin ${ }^{1}$, Wen Gao ${ }^{1}$, Shuangjing Chen ${ }^{2}$, Renhua Guo ${ }^{1}$ \\ ${ }^{1}$ Department of Medical Oncology, the First Affiliated Hospital of Nanjing Medical University, Nanjing, China; ${ }^{2}$ Institute of Occupational Disease \\ Prevention, Jiangsu Provincial Center for Disease Prevention and Control, Nanjing, China \\ Contributions: (I) Conception and design: C Zhang, F Gao; (II) Administrative support: R Guo; (III) Provision of study materials or patients: R Guo, S \\ Jin, W Gao; (IV) Collection and assembly of data: C Zhang, F Gao, S Chen; (V) Data analysis and interpretation: C Zhang, F Gao; (VI) Manuscript \\ writing: All authors; (VII) Final approval of manuscript: All authors. \\ \#These authors contributed equally to this work. \\ Correspondence to: Renhua Guo. Department of Medical Oncology, the First Affiliated Hospital of Nanjing Medical University, 300 Guangzhou Road, \\ Nanjing, China. Email: rhguo@njmu.edu.cn.
}

\begin{abstract}
Background: Checkpoint inhibitor pneumonitis (CIP) may be accompanied by lung cancer in patients treated with immune checkpoint inhibitors (ICI). This study aimed to test the risk factors, genetic and clinical characteristics of CIP in a cohort of Chinese patients with lung cancer.

Methods: We retrospectively reviewed the medical records of eligible patients who received ICI treatment from December 2017 to September 2020 in our hospital. Patient characteristics, ICI protocols, and mutation frequencies of related genes are compared between the CIP group and the non-CIP group.

Results: A total of 94 patients were recruited. Of them, 16 (17.0\%) patients developed CIP. Multivariate logistic regression analysis suggested Eastern Cooperative Oncology Group (ECOG) performance status $(\mathrm{PS}) \geq 2$ [odds ratio $(\mathrm{OR})=6.53 ; 95 \%$ confidence interval $(\mathrm{CI}), 1.74-24.46 ; \mathrm{P}=0.005$ ] and previous pulmonary fibrosis (OR =20.13; 95\% CI, 3.64-111.44; P=0.001) were independently associated with a higher incidence of CIP. There was an increasing trend, although not statistically significant, in the risk of CIP in patients with TP53 mutation $(\mathrm{P}=0.280)$. Most CIP patients were managed successfully following the current guideline. However, serious events (including one death) were still observed.

Conclusions: ECOG PS $\geq 2$ and earlier pulmonary fibrosis were closely correlated to the occurrence of CIP in Chinese lung cancer patients after ICI treatment. Early screening and prompt intervention are necessary for the management of CIP.
\end{abstract}

Keywords: Immune checkpoint inhibitor (ICI); pneumonitis; risk factors

Submitted Aug 25, 2020. Accepted for publication Oct 30, 2020.

doi: 10.21037/apm-20-1823

View this article at: http://dx.doi.org/10.21037/apm-20-1823

\section{Introduction}

Immune checkpoint inhibitors (ICI), including anticytotoxic T lymphocyte antigen 4 (CTLA-4), antiprogrammed cell death 1 (PD-1), and anti-programmed cell death 1 ligand 1 (PD-L1) antibodies, might be a significant oncological breakthrough over the past decade and have reshaped the treatment paradigms in various malignancies. Clinical trials have shown the superiority of ICI over traditional strategies in dealing with non- small cell lung cancer (NSCLC) (1-5) and small cell lung cancer (SCLC) (6). As suggested in many clinical trials, ICI is less toxic than standard chemotherapy. However, some patients treated with ICI may still develop severe, potentially life-threatening immune-related adverse events (irAE), including pneumonitis, termed checkpoint inhibitor pneumonitis (CIP).

The reported incidence of CIP may rise to $5 \%$ (7), even higher in lung cancer patients (8). As an uncommon but 
potentially severe complication, CIP typically presents with dyspnea, cough, fever, and chest pain along with pulmonary infiltrates on chest imaging (9). Several pieces of recent literature have reported the risk factors of CIP in patients treated with ICI. However, similar reports in Chinese lung cancer patients are scarce. Despite this, Asians are more susceptible to pneumonitis, as shown by the higher incidence of pneumonitis in Japan (10). The underlying association between genetic polymorphisms and CIP susceptibility deserves to be explored.

Therefore, we designed this study to describe the clinical course, genetic and radiologic characteristics, and risk factors of CIP in Chinese lung cancer patients.

We present the following article in accordance with the STROBE reporting checklist (available at http://dx.doi. org/10.21037/apm-20-1823).

\section{Methods}

\section{Study population and data collection}

We performed a retrospective study on the patients confirmed with lung cancer and treated with ICI at the First Affiliated Hospital of Nanjing Medical University between December 2017 and September 2020. Excluded were those patients who received a placebo or undetermined therapy, or a follow-up period (between the first ICI exposure and the latest follow-up) of less than 21 days.

Finally, we recruited 94 patients and collected their medical records to present related characteristics, including age, gender, smoking history, Eastern Cooperative Oncology Group (ECOG) performance status (PS), tumor histologic type, extrathoracic metastasis, treatment line, previous thoracic radiotherapy, and treatment regimen. We acquired somatic mutations data of 57 patients through next-generation sequencing (NGS). All detectable genes are detailed in Table S1. Because of insufficient PD-L1 expression information of a significant fraction of patients, PD-L1 was not analyzed in this study.

\section{Diagnosis, assessment, and management of CIP}

The diagnosis of CIP was established on the results of chest computed tomography (CT) and clinical findings after the exclusion of other known etiologies, including infection, tumor progression, congestive heart failure, etc. Invasive diagnostic measures were not taken after a careful risk-benefit analysis. The severity of CIP was assessed following the Common Terminology Criteria for Adverse Events (version 5.0). The attending physician determined the clinical intervention of CIP according to the recommendations of the American Society of Clinical Oncology Clinical Practice Guideline (11).

\section{Radiological analysis and response evaluation}

As recommended by the American Society of Clinical Oncology Clinical Practice Guideline, the diagnosis of CIP was suspected once focal or diffuse inflammation of the lung parenchyma (typically identified on CT imaging) was discovered. After clinical confirmation, the radiology findings, including reticulation, honeycombing, traction, bronchiectasis, ground-glass opacity (GGO), were classified with the five distinct radiologic subtypes of CIP reported by Naidoo et al. (9), as (I) cryptogenic organizing pneumonia (COP)-like, (II) GGO, (III) hypersensitivity type, (IV) interstitial type, and (V) pneumonitis not otherwise specified. Representative CT images of common phenotypes of CIP are shown in Figure 1.

Diagnostic imaging evaluated treatment response following the Response Evaluation Criteria in Solid Tumors (RECIST) group criteria version 1.1. The maximal tumor shrinkage (MTS) was defined as the highest tumor shrinkage rate compared to baseline during treatment with ICI. Progression-free survival (PFS) was defined as time from start of ICI treatment until the first observed progression. Overall survival (OS) was defined as time from start of ICI treatment until death.

\section{Statistical analysis}

All patients were classified into two groups with the occurrence of CIP. The student's $t$-test was used for continuous variables and Pearson's chi-square test or Fisher's exact test for discrete variables. The univariate and multivariate logistic regression model analyzed the association between the development of CIP. The MTS was compared using the Mann-Whitney test. PFSs were estimated with the Kaplan-Meier method and compared with the $\log$-rank test. A P value $<0.05$ was considered to show statistical significance. All analyses were conducted with SPSS 23.0 and GraphPad Prism 8.3.0.

\section{Study ethics}

The study was approved by the ethics committees of the 

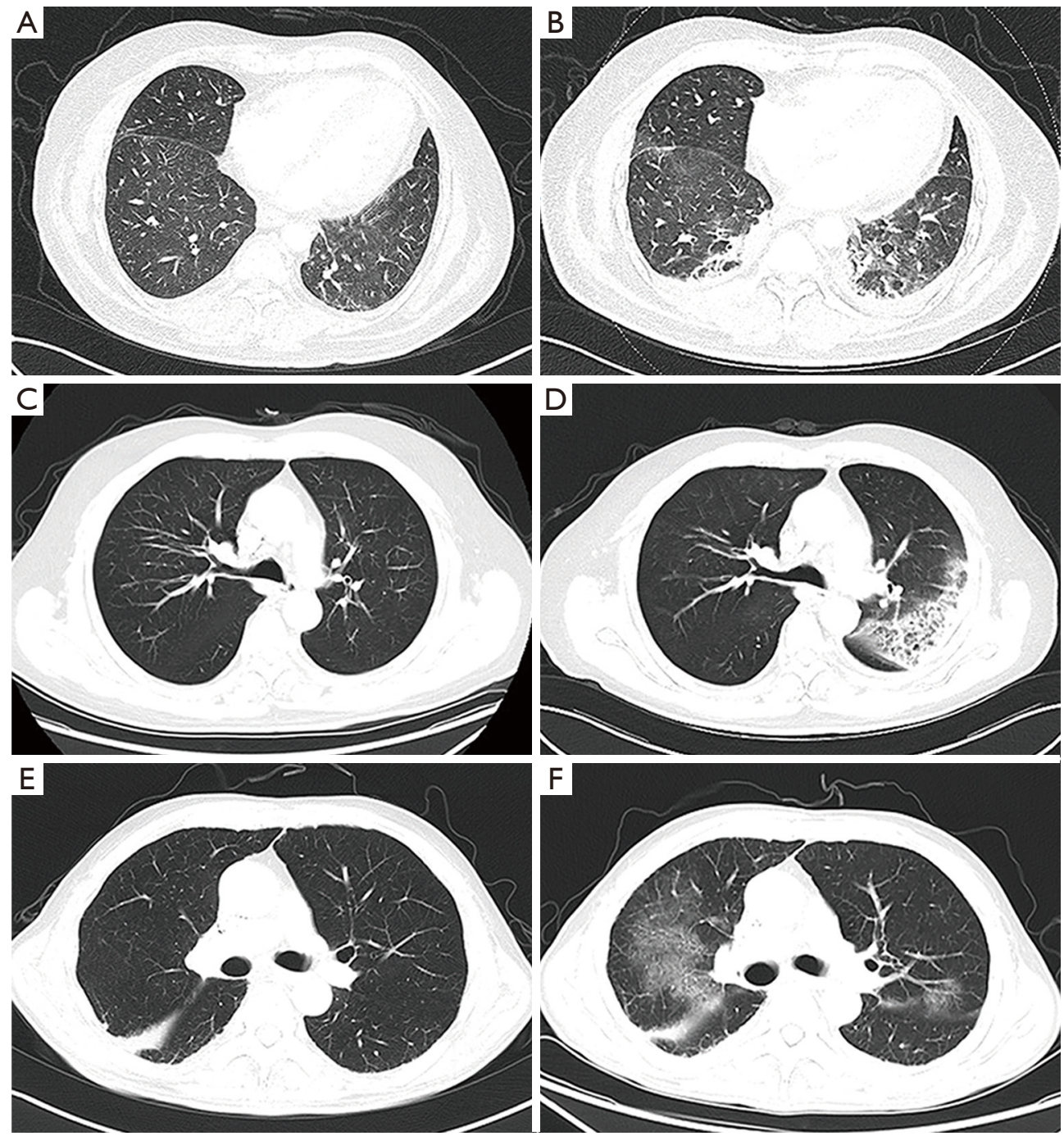

Figure 1 Radiological features of checkpoint inhibitor pneumonitis with common phenotypes. (A,B) Baseline CT images and pneumonitis with a COP-like subtype in a 46-year-old male treated with nivolumab. (C,D) Baseline CT images and pneumonitis with an interstitial subtype in a 65-year-old male treated with nivolumab. (E,F) Baseline CT images and pneumonitis with a GGO subtype in a 68-year-old male treated with nivolumab. COP, cryptogenic organizing pneumonia; GGO, ground-glass opacity; CT, computed tomography.

First Affiliated Hospital of Nanjing Medical University (No. 2019-SRFA-226). All procedures performed in this study involving human participants were in accordance with the Declaration of Helsinki (as revised in 2013). Individual consent for this retrospective analysis was waived.

\section{Results}

\section{Patients' characteristics}

We recruited 94 patients with lung cancer who received ICI treatment. The $16 \mathrm{CIP}$ cases $(17.0 \%)$ were assigned to the CIP group, and the left 78 cases to the non-CIP group. The follow-up period (median, 262 vs. 314 days; $\mathrm{P}=0.410$ ) and the number of ICI exposures (median, 13 vs. 10; $\mathrm{P}=0.410$ ) showed no difference between the two groups at the time of data cutoff. We summarized the baseline characteristics and immunotherapy protocols in Table 1. Of the 94 patients, non-small lung cancer was the predominant histologic type, but there were also several small cell lung cancer patients in both groups (12.5\% in the CIP group vs. $12.8 \%$ in the non- 
Table 1 Patient characteristics and immunotherapy protocols $(\mathrm{N}=94)$

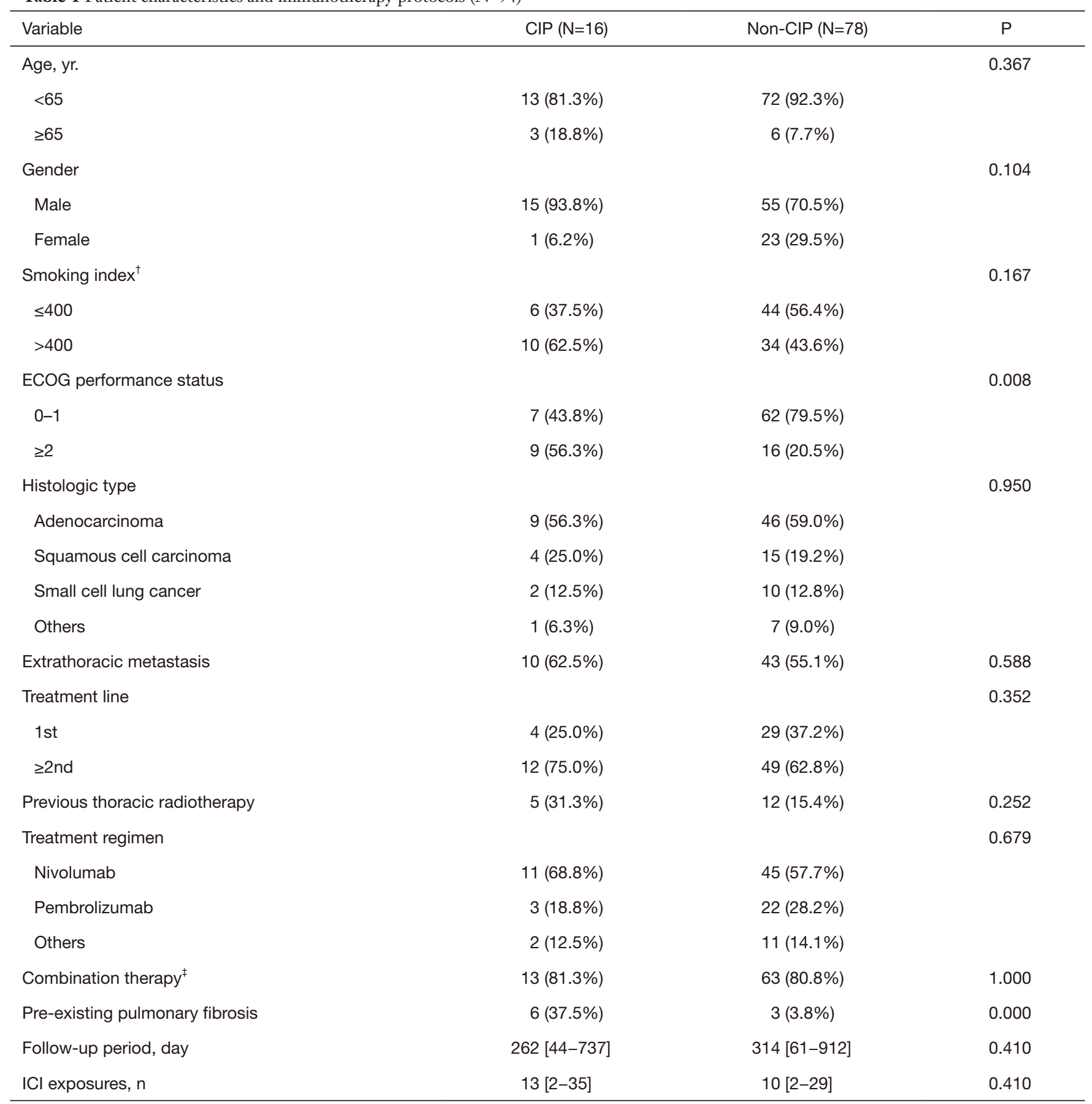

${ }^{\dagger}$, smoke index was calculated as years of smoking multiplied by cigarettes per day; ${ }^{\ddagger}$, combination therapy was determined as immunotherapy combined with chemotherapy or anti-angiogenic therapy. CIP, checkpoint inhibitor pneumonitis; ECOG, Eastern Cooperative Oncology Group; ICl, immune-checkpoint inhibitor.

CIP group). As shown in Table 1, no significant differences were observed in the baseline characteristics, except for ECOG PS and pre-existing pulmonary fibrosis that were more prevalent in the CIP group: ECOG PS $\geq 2(56.3 \%$ vs. $20.5 \% ; \mathrm{P}=0.008)$ and pre-existing pulmonary fibrosis $(37.5 \%$ vs. $3.8 \% ; \mathrm{P}=0.000)$. 
Table 2 Risk factors of checkpoint inhibitor pneumonitis

\begin{tabular}{|c|c|c|c|c|c|c|}
\hline Risk factor & \multicolumn{3}{|c|}{ Univariate } & \multicolumn{3}{|c|}{ Multivariate } \\
\hline ECOG performance status $\geq 2$ & 4.98 & $(1.61-15.43)$ & 0.005 & 6.53 & $(1.74-24.46)$ & 0.005 \\
\hline Pre-existing pulmonary fibrosis & 15.00 & (3.23-69.62) & 0.001 & 20.13 & (3.64-111.44) & 0.001 \\
\hline
\end{tabular}

ECOG, Eastern Cooperative Oncology Group; OR, odds ratio; Cl, confidence interval.

Table 3 Genetic characteristics of checkpoint inhibitor pneumonitis $(\mathrm{N}=57)$

\begin{tabular}{llcc}
\hline Mutation & CIP $(\mathrm{N}=11)$ & Non-CIP $(\mathrm{N}=46)$ & $\mathrm{P}$ \\
\hline TP53 & $9(81.8 \%)$ & $27(58.7 \%)$ & 0.280 \\
KRAS & $3(27.3 \%)$ & $7(15.2 \%)$ & 0.615 \\
EGFR & $2(18.2 \%)$ & $7(15.2 \%)$ & 1.000 \\
DPYD & $1(9.1 \%)$ & $8(17.4 \%)$ & 0.827 \\
NQO1 & $2(18.2 \%)$ & $7(15.2 \%)$ & 1.000 \\
LRP1B & $1(9.1 \%)$ & $7(15.2 \%)$ & 0.966 \\
SMARCA4 & $1(9.1 \%)$ & $7(15.2 \%)$ & 0.966 \\
PTEN & $2(18.2 \%)$ & $4(8.7 \%)$ & 0.708 \\
ERCC1 & $0(0.0 \%)$ & $6(13.0 \%)$ & 0.472 \\
FAT1 & $1(9.1 \%)$ & $5(10.9 \%)$ & 1.000 \\
\hline
\end{tabular}

CIP, checkpoint inhibitor pneumonitis.

\section{Risk factors of CIP}

Univariate analysis found that ECOG PS $\geq 2(\mathrm{OR}=4.98$; 95\% CI, 1.61-15.43; $\mathrm{P}=0.005)$ and pre-existing pulmonary fibrosis (OR =15.00; 95\% CI, 3.23-69.62; $\mathrm{P}=0.001$; Table 2) were two risk factors of CIP. Then, the two factors were subjected to multivariate analysis, finding that poor ECOG PS (OR $=6.53 ; 95 \%$ CI, 1.74-24.46; $\mathrm{P}=0.005)$ and baseline radiographic showed pulmonary fibrosis $(\mathrm{OR}=20.13 ; 95 \%$ CI, 3.64-111.44; $\mathrm{P}=0.001$; Table 2) were independently associated with a higher incidence of CIP.

To explore the genetic factors predicting the development of CIP, we performed somatic mutation analysis in 57 patients whose NGS data were available. The top ten most often mutated genes were screened out (detailed information in Table S2), with the highest mutation frequency in TP53 $(n=36 ; 63.2 \%$; Table 3). However, both groups showed similar somatic mutation profiles. An increasing trend in CIP risk was observed in patients with TP53 mutation, but this increase showed no between-group difference $(\mathrm{P}=0.280)$.

\section{Clinical and radiological features of CIP}

We detail the clinical courses and radiological features of the CIP group in Table 4. The median onset time of CIP was 90 days (range, 22-326 days). The CIP was classified as grade 1 in 3 patients (18.8\%), Grade 2 in 9 patients $(56.3 \%)$, grade 3 in 4 patients $(25.0 \%)$, and none as grade 4. The most common initial symptom was dyspnea $(\mathrm{n}=11$; $68.8 \%)$, followed by cough $(\mathrm{n}=8 ; 50.0 \%)$ and fever $(\mathrm{n}=3$; $18.8 \%)$. Three $(18.8 \%)$ patients were asymptomatic. The radiological characteristics of CIP were represented by COP-like type $(\mathrm{n}=6 ; 37.5 \%)$, interstitial type $(\mathrm{n}=5 ; 31.3 \%)$, GGO ( $\mathrm{n}=4 ; 25.0 \%)$, and pneumonitis not otherwise specified $(\mathrm{n}=1 ; 6.3 \%)$. Eight $(50.0 \%)$ patients developed concurrent irAE, including 3 cases of thyroid dysfunction, 2 cases of skin rash, 1 case of myocarditis, 1 case of hepatitis and 1 case of enteritis.

Among the 13 patients with CIP, 13 (81.3\%) were administered with corticosteroids as per the current guidelines. Four patients were empirically treated with antibiotics. During the follow-up, the majority $(n=12$; $75.0 \%$ ) underwent CIP improvement or complete recovery. One patient dropped out of the follow-up, and one died of intestinal perforation arising from concurrent checkpoint inhibitor-related enteritis. After clinical and radiographic improvement, nine patients with CIP received an ICI rechallenge at the discretion of the attending physician. Recurrent CIP was noted in six $(66.7 \%)$ cases, and their ICI treatment was stopped permanently.

\section{Effect of CIP on ICI outcomes}

Next, we assessed the impact of CIP on clinical outcomes of ICI treatment. The objective response rate was similar between both groups (43.8\% vs. 24.4\%; $\mathrm{P}=0.203$ ). Although the difference was not statistically, we observed a slightly higher disease control rate in the CIP group $(100.0 \% v s$. $91.0 \% ; \mathrm{P}=0.470)$. Greater MTS rate was noted in patients who experienced CIP during ICI treatment (median, 25.5\% 
Table 4 The clinical course of checkpoint inhibitor pneumonitis $(\mathrm{N}=16)$

\begin{tabular}{|c|c|}
\hline Variable & $\begin{array}{c}\mathrm{ICl} \text {-related } \\
\text { pneumonitis }(\mathrm{N}=16)\end{array}$ \\
\hline Drug exposures, $\mathrm{n}$ & $5[1-16]$ \\
\hline Time to pneumonitis, days & 90 [22-326] \\
\hline \multicolumn{2}{|l|}{ Grade } \\
\hline Grade 1 & $3(18.8 \%)$ \\
\hline Grade 2 & $9(56.3 \%)$ \\
\hline Grade 3 & $4(25.0 \%)$ \\
\hline \multicolumn{2}{|l|}{ Initial manifestation } \\
\hline Dyspnea & $11(68.8 \%)$ \\
\hline Cough & $8(50.0 \%)$ \\
\hline Fever & $3(18.8 \%)$ \\
\hline Asymptomatic & $3(18.8 \%)$ \\
\hline \multicolumn{2}{|l|}{ Radiological phenotypes } \\
\hline COP & $6(37.5 \%)$ \\
\hline Interstitial & $5(31.3 \%)$ \\
\hline GGO & $4(25.0 \%)$ \\
\hline Pneumonitis not otherwise specified & $1(6.3 \%)$ \\
\hline \multicolumn{2}{|l|}{ Concurrent irAE } \\
\hline Thyroid dysfunction & $3(18.8 \%)$ \\
\hline Skin rash & $2(12.5 \%)$ \\
\hline Myocarditis & $1(6.3 \%)$ \\
\hline Hepatitis & $1(6.3 \%)$ \\
\hline Enteritis & $1(6.3 \%)$ \\
\hline $\mathrm{ICl}$ discontinuation & $12(75.0 \%)$ \\
\hline \multicolumn{2}{|l|}{ Treatment } \\
\hline Steroid & $13(81.3 \%)$ \\
\hline Antibiotics & $4(25.0 \%)$ \\
\hline \multicolumn{2}{|l|}{ Outcome } \\
\hline Complete recovery & $7(43.8 \%)$ \\
\hline Improved & $5(31.3 \%)$ \\
\hline Stable & $2(12.5 \%)$ \\
\hline Death & $1(6.3 \%)$ \\
\hline Unknown & $1(6.3 \%)$ \\
\hline ICI re-challenge & 9 (56.3\%) \\
\hline
\end{tabular}

$\mathrm{ICl}$, immune-checkpoint inhibitor; COP, cryptogenic organizing pneumonia; GGO, ground-glass opacity; irAE, immune-related adverse events. vs. $0.0 \%, \mathrm{P}=0.014$; Figure $2 A, \mathrm{~B})$. The median PFS of all patients was $496 \pm 101$ days (Figure $2 C$ ). In the subgroup analysis, PFS tended to be longer in the CIP group than in the non-CIP group (not reached vs. $412 \pm 79$ days, $\mathrm{P}=0.493$; Figure 2D), though this difference was not statistically significant. OS was not mature at the data cutoff, and the impact on OS was not estimated.

\section{Discussion}

We here reported the characteristics and risk factors of CIP in a cohort of Chinese patients with ICI-treated lung cancer. In the present study, the incidence of CIP was $17.0 \%$. ECOG PS $\geq 2(\mathrm{OR}=6.53 ; 95 \%$ CI, $1.74-24.46 ; \mathrm{P}=0.005)$ and pre-existing pulmonary fibrosis $(\mathrm{OR}=20.13$; $95 \% \mathrm{CI}$, 3.64-111.44; $\mathrm{P}=0.001$; Table 2) were associated with the higher incidence of CIP, while other factors [including extrathoracic metastasis, smoking history, previous thoracic radiotherapy, combination therapy (12-15)] were not. We also compared the incidence of CIP among patients treated with different ICI. However, the risk of CIP did not differ among patients treated with nivolumab, pembrolizumab or other candidate ICI used in clinical trials $(\mathrm{P}=0.679)$, which coincided with previous study (16).

Consistent with earlier studies, our logistic regression analyses showed that patients with poor ECOG PS were more susceptible to developing CIP (17). Studies indicated a higher ECOG PS strongly correlated with worse survival after ICI treatment $(18,19)$. Therefore, ICI clinical trials recruited, with rare exceptions, only patients with ECOG PS 0 or 1 (1-6). However, in our analysis, $26.6 \%$ of the patients showed ECOG PS $\geq 2$, offering a more reliable explanation for the higher incidence of CIP than earlier studies (7). This finding reminds clinicians of the possibility of CIP when treating lung cancer patients with a high ECOG score.

Another key exclusion criterion in ICI clinical trials is an interstitial lung disease. Patients with interstitial lung disease may also benefit from ICI treatment (20). Of the nine patients with pulmonary fibrosis, six developed CIP, including two who had been diagnosed with interstitial lung disease. This finding shows that pre-existing pulmonary fibrosis is strongly related to the occurrence of CIP $(12,21)$. The factors that predispose patients with pulmonary fibrosis to CIP have not been fully explained. Given the evidence for the similarity in the alveolar immune cell landscape between patients with CIP and patients with interstitial lung disease (22), we speculate that it may involve the analogous 

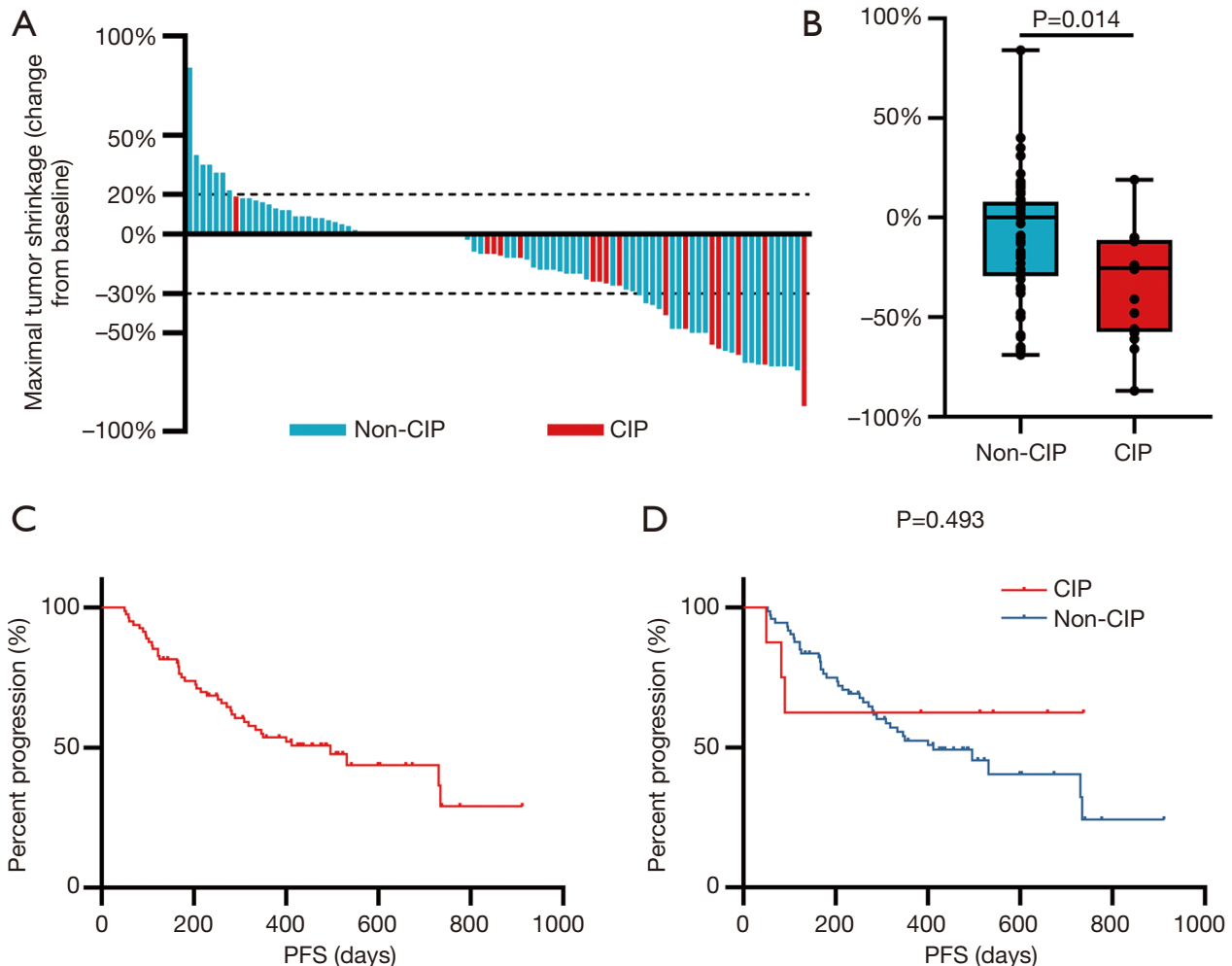

Figure 2 Correlation between checkpoint inhibitor pneumonitis development and clinical outcomes during ICI treatment. (A,B) The MTS rate revealed a deeper remission in the CIP group during ICI treatment (median, $25.5 \%$ vs. 0.0\%, P=0.014). (C) The median PFS for all patients was $496 \pm 101$ days. (D) PFS tended to be longer in the CIP group (not reached) than in the non-CIP group (412 \pm 79 days, $\mathrm{P}=0.493$ ). MTS, The maximal tumor shrinkage; CIP, checkpoint inhibitor pneumonitis; ICI, immune checkpoint inhibitors; PFS, progression-free survival.

alveolar immune cell landscape in the pathogenesis of CIP. Hence, the bronchoalveolar lavage may be recommended before ICI administration.

Studies concerning genetic characteristics of CIP are scarce. Owen et al. (23) reported fewer irAE developed in patients harboring KRAS and TP53 mutation. However, our findings were contrary. The rate of TP53 mutation, the most frequent in the total population, was higher in the CIP group ( $81.8 \%$ vs. $58.7 \%$; $\mathrm{P}=0.280)$. A similar difference was identified in KRAS (27.3\% vs. $15.2 \%$; $\mathrm{P}=0.615)$, though not statistically obvious. We concluded that TP53 and KRAS mutations might increase a patient's susceptibility to CIP. However, we focused only on somatic mutations in our analysis, further studies should be performed to analyze genetic characteristics in detail.

The higher CIP incidence we observed in the present study may be attributed to our actual clinical setting. Also, irAEs, including CIP, has gained increasing attention by clinicians. With stricter management of ICI-related adverse events, more CIP cases may be reported, causing its incidence higher than before. Similar to previous study $(9,15)$, the median onset time of CIP was 90 days (range, 22-326 days). This result indicates that CIP may occur early or late after ICI treatment. Suresh et al. (15) also proposed two different phenotypes of CIP: early-onset CIP and lateonset CIP. These data support the crucial need to carefully monitor CIP during the full course of immunotherapy. When monitoring this adverse event, the clinicians should raise awareness of the typical CIP symptoms, including dyspnea and cough. CT is recommended to provide clues of CIP (11). The diagnosis of CIP was suspected once focal or diffuse inflammation of the lung parenchyma was discovered after the ICI treatment. Among the 16 patients with CIP, COP-like was the most common radiological phenotype. Patients of this type may respond better to glucocorticoid therapy (24). In the present study, most CIP patients were managed successfully. Only one patient died of intestinal perforation caused by concurrent immunerelated enteritis, which reminds clinicians to pay attention to the simultaneous organ immune adverse events. In our 
study, nine patients restarted ICI therapy, and six of them experienced CIP relapse, suggestive of the necessity of patient-screening before ICI treatment.

The relationship between the irAE and prognosis of ICItreated patients has been repeatedly reported. Haratani et al. (25) pointed out that the prognosis of nivolumab treatment was positively correlated with the occurrence of irAE, supported by many other studies $(23,26,27)$. In our analysis, we observed no increased objective response rate in patients with CIP. Then we further tested the response by comparing the MTS rate between the two groups, finding that the CIP group showed deeper remission (median, $25.5 \%$ vs. $0.0 \%, \mathrm{P}=0.014$ ). The estimated median PFS is longer in CIP group (not reached) than in non-CIP group (412 \pm 79 days), though not statistically significant $(\mathrm{P}=0.493)$. We expect larger sample size could bring more credible results. We further performed subgroup analysis by different ICI. Consistent with prior study (16), there was no significant difference in PFS among patients treated with different ICI ( $\mathrm{P}=0.857$; data not shown).

There are several limitations to our study. First, it is a single-center, retrospective study with small sample size, increasing the risk of patient selection bias and group selection bias. Prospective studies and larger-size analysis will be needed to verify these results. A second limitation is insufficient invasive means to confirm the diagnosis of CIP, which may be an obstacle in exploring the pathogenesis of CIP. Third, our analysis of genetic characteristics is not comprehensive. We will further investigate the possible association of genetic polymorphisms and CIP susceptibility by performing genome-wide association study in the future.

In conclusion, our study identified ECOG PS $\geq 2$ and previous pulmonary fibrosis are closely correlated to the occurrence of CIP in Chinese lung cancer patients after ICI treatment, a finding that causes early screening and timely intervention necessary in the management of CIP.

\section{Acknowledgments}

Funding: None.

\section{Footnote}

Reporting Checklist: The authors have completed the STROBE reporting checklist. Available at http://dx.doi. org/10.21037/apm-20-1823

Data Sharing Statement: Available at http://dx.doi. org/10.21037/apm-20-1823
Conflicts of Interest: All authors have completed the ICMJE uniform disclosure form (available at http://dx.doi. org/10.21037/apm-20-1823). The authors have no conflicts of interest to declare.

Ethical Statement: The authors are accountable for all aspects of the work in ensuring that questions related to the accuracy or integrity of any part of the work are appropriately investigated and resolved. The study was approved by the ethics committees of the First Affiliated Hospital of Nanjing Medical University (No. 2019-SRFA226). All procedures performed in this study involving human participants were in accordance with the Declaration of Helsinki (as revised in 2013). Individual consent for this retrospective analysis was waived.

Open Access Statement: This is an Open Access article distributed in accordance with the Creative Commons Attribution-NonCommercial-NoDerivs 4.0 International License (CC BY-NC-ND 4.0), which permits the noncommercial replication and distribution of the article with the strict proviso that no changes or edits are made and the original work is properly cited (including links to both the formal publication through the relevant DOI and the license). See: https://creativecommons.org/licenses/by-nc-nd/4.0/.

\section{References}

1. Borghaei H, Paz-Ares L, Horn L, et al. Nivolumab versus Docetaxel in Advanced Nonsquamous Non-Small-Cell Lung Cancer. N Engl J Med 2015;373:1627-39.

2. Metro G, Addeo A, Signorelli D, et al. Outcomes from salvage chemotherapy or pembrolizumab beyond progression with or without local ablative therapies for advanced non-small cell lung cancers with PD-L1 50\% who progress on first-line immunotherapy: real-world data from a European cohort. J Thorac Dis 2019;11:4972-81.

3. Hellmann MD, Ciuleanu TE, Pluzanski A, et al. Nivolumab plus Ipilimumab in Lung Cancer with a High Tumor Mutational Burden. N Engl J Med 2018;378:2093-104.

4. Herbst RS, Baas P, Kim DW, et al. Pembrolizumab versus docetaxel for previously treated, PD-L1-positive, advanced non-small-cell lung cancer (KEYNOTE-010): a randomised controlled trial. Lancet 2016;387:1540-50.

5. Travert C, Tomasini P, Jeanson A, et al. Firstline pembrolizumab in programmed death ligand 1 positive non-small cell lung cancer. Transl Cancer Res 2019;8:2514-6. 
6. Paz-Ares L, Dvorkin M, Chen Y, et al. Durvalumab plus platinum-etoposide versus platinum-etoposide in firstline treatment of extensive-stage small-cell lung cancer (CASPIAN): a randomised, controlled, open-label, phase 3 trial. Lancet 2019;394:1929-39.

7. Martins F, Sofiya L, Sykiotis GP, et al. Adverse effects of immune-checkpoint inhibitors: epidemiology, management and surveillance. Nat Rev Clin Oncol 2019;16:563-80.

8. Nishino M, Giobbie-Hurder A, Hatabu H, et al. Incidence of Programmed Cell Death 1 InhibitorRelated Pneumonitis in Patients With Advanced Cancer: A Systematic Review and Meta-analysis. JAMA Oncol 2016;2:1607-16.

9. Naidoo J, Wang X, Woo KM, et al. Pneumonitis in Patients Treated With Anti-Programmed Death-1/Programmed Death Ligand 1 Therapy. J Clin Oncol 2017;35:709-17.

10. Kudoh S, Kato H, Nishiwaki Y, et al. Interstitial lung disease in Japanese patients with lung cancer: a cohort and nested case-control study. Am J Respir Crit Care Med 2008; 177:1348-57.

11. Brahmer JR, Lacchetti C, Schneider BJ, et al. Management of Immune-Related Adverse Events in Patients Treated With Immune Checkpoint Inhibitor Therapy: American Society of Clinical Oncology Clinical Practice Guideline. J Clin Oncol 2018;36:1714-68.

12. Cho JY, Kim J, Lee JS, et al. Characteristics, incidence, and risk factors of immune checkpoint inhibitor-related pneumonitis in patients with non-small cell lung cancer. Lung Cancer 2018;125:150-56.

13. Dávila-Dupont D, Motola-Kuba D, Dorantes-Heredia $\mathrm{R}$, et al. Risk of Pneumonitis with the Use of Different Immune Checkpoint Inhibitors in a Mexican Population. Oncology 2019;96:268-72.

14. Voong KR, Hazell SZ, Fu W, et al. Relationship Between Prior Radiotherapy and Checkpoint-Inhibitor Pneumonitis in Patients With Advanced Non-Small-Cell Lung Cancer. Clin Lung Cancer 2019;20:e470-9.

15. Suresh K, Voong KR, Shankar B, et al. Pneumonitis in Non-Small Cell Lung Cancer Patients Receiving Immune Checkpoint Immunotherapy: Incidence and Risk Factors. J Thorac Oncol 2018;13:1930-9.

16. Passiglia F, Galvano A, Rizzo S, et al. Looking for the best immune-checkpoint inhibitor in pre-treated NSCLC patients: An indirect comparison between nivolumab, pembrolizumab and atezolizumab. Int J Cancer 2018;142:1277-84.

17. Tone M, Izumo T, Awano N, et al. High mortality and poor treatment efficacy of immune checkpoint inhibitors in patients with severe grade checkpoint inhibitor pneumonitis in non-small cell lung cancer. Thorac Cancer 2019;10:2006-12.

18. Tournoy KG, Thomeer M, Germonpre P, et al. Does nivolumab for progressed metastatic lung cancer fulfill its promises? An efficacy and safety analysis in 20 general hospitals. Lung Cancer 2018;115:49-55.

19. Huemer F, Lang D, Westphal T, et al. Baseline Absolute Lymphocyte Count and ECOG Performance Score Are Associated with Survival in Advanced Non-Small Cell Lung Cancer Undergoing PD-1/PD-L1 Blockade. J Clin Med 2019;8:1014.

20. Ide M, Tanaka K, Sunami S, et al. Durable response to nivolumab in a lung adenocarcinoma patient with idiopathic pulmonary fibrosis. Thorac Cancer 2018;9:1519-21.

21. Yamaguchi T, Shimizu J, Hasegawa T, et al. Pre-existing pulmonary fibrosis is a risk factor for anti-PD-1-related pneumonitis in patients with non-small cell lung cancer: A retrospective analysis. Lung Cancer 2018;125:212-17.

22. Suresh K, Naidoo J, Zhong Q, et al. The alveolar immune cell landscape is dysregulated in checkpoint inhibitor pneumonitis. J Clin Invest 2019;129:4305-15.

23. Owen DH, Wei L, Bertino EM, et al. Incidence, Risk Factors, and Effect on Survival of Immune-related Adverse Events in Patients With Non-Small-cell Lung Cancer. Clin Lung Cancer 2018;19:e893-900.

24. Nagata N, Nagatomo H, Yoshii C, et al. Features of idiopathic pulmonary fibrosis with organizing pneumonia. Respiration 1997;64:331-5.

25. Haratani K, Hayashi H, Chiba Y, et al. Association of Immune-Related Adverse Events With Nivolumab Efficacy in Non-Small-Cell Lung Cancer. JAMA Oncology 2018;4:374-378.

26. Freeman-Keller M, Kim Y, Cronin H, et al. Nivolumab in Resected and Unresectable Metastatic Melanoma: Characteristics of Immune-Related Adverse Events and Association with Outcomes. Clin Cancer Res 2016;22:886-94.

27. Kimbara S, Fujiwara Y, Iwama S, et al. Association of antithyroglobulin antibodies with the development of thyroid dysfunction induced by nivolumab. Cancer Sci 2018;109:3583-90.

Cite this article as: Zhang C, Gao F, Jin S, Gao W, Chen S, Guo R. Checkpoint inhibitor pneumonitis in Chinese lung cancer patients: clinical characteristics and risk factors. Ann Palliat Med 2020;9(6):3957-3965. doi: 10.21037/apm-20-1823 